\title{
Nonlinear moving horizon estimator for online estimation of the density and viscosity of a
} mineral slurry

\author{
Jenny L. Diaz C., ${ }^{*,+} \ddagger$ Carlos Ocampo-Martinez, ${ }^{\dagger}$ and Hernan Alvarez ${ }^{\ddagger}$ \\ +Universitat Politècnica de Catalunya, Institut de Robòtica i Informàtica Industrial (CSIC-UPC), \\ Llorens i Artigas 4-6, Planta 2, 08028 Barcelona, Spain \\ $\ddagger K A L M A N$, Grupo de Investigación en Procesos Dinámicos, Universidad Nacional de \\ Colombia-Sede Medellín, Facultad de Minas. Cra 80 No. 65-223, Medellín, Colombia \\ E-mail: jdiaz@iri.upc.edu,jldiazc@unal.edu.co
}

\begin{abstract}
This paper proposes a moving horizon estimator for nonlinear systems with unknown inputs, which do not comply with the model structures proposed in the literature for the design of nonlinear observers. The estimator is designed as an optimization problem over a moving horizon, constrained to process model equations and considering the unknown inputs as random inputs among their operating bounds. This proposal is applied to the transport of mineral slurries among process units, typically present in chemical and biological processes. There, to have the slurry properties as on-line measurements is vital to an efficient control of those processing units. The performance of proposed estimator is evaluated by simulation with data from a real processing plant, and its performance is compared with a linear estimator executing the same estimation task. Better results are obtained using the proposed estimator by considering the nonlinearities of the process.
\end{abstract}




\section{Introduction}

Nonlinear systems have a wide application in chemical and biological processes because the complexity of phenomena that govern these sort of processes, which are not usually linearized. Thus, from the nonlinear systems, it is possible to represent the main phenomena that govern the behavior of these systems. However, nonlinear models are quite restrictive for designing observers due to the particularities of each process and their nonlinear terms in the model equations. ${ }^{1}$ Therefore, the study of the nonlinear dynamics, the modeling process, and the development of new control strategies correspond to an active research area, with important developments in the science, the engineering, and other fields in the last decades. ${ }^{2}$ Nevertheless, these processes, as well as linear systems, have the problem of needing internal information of the process from their available measurements. This necessity arises when tasks such as modeling, monitoring, and/or control are required. ${ }^{3}$

In the minerals processing industry, the particulate minerals are usually mixed with water in order to ease their transport and benefit. This mixture is known as mineral slurry. Slurry properties like density and viscosity are strongly affected by solid phase concentration. In addition, these properties are normally used as the quality indicator of the processed minerals, and for determining the energy efficiency of the process. Therefore, the slurry properties must be correctly determined, measured, or estimated for guaranteeing the optimal operating conditions. However, and due to the presence of solids, the mixture properties are difficult to measure online and must be estimated by using observers based on models. When the observers are designed based on a phenomenological model, fact that allows modeling the phenomena in the process, in some cases the nonlinearities of the model equations cannot be organized in the suggested form as the proposed observer structures in the literature. ${ }^{1}$ Therefore, observers able to estimate the slurry properties for the transport processes, which in addition considered the disturbances and the nonlinearities, are required for completing the implemented monitoring and control systems. ${ }^{4}$

To obtain the variables estimation of the nonlinear dynamics, many approaches have 
been developed. Among them, it can be mentioned the Extended Kalman filter (EKF), the $H_{\infty}$ observer, ${ }^{5}$ observers designed using linear matrix inequalities, ${ }^{6}$ or sliding mode observers for uncertain systems. ${ }^{7}$ Nevertheless, most of these designs are quite restrictive regarding the model structure and their nonlinear functions. To overcome this fact, estimation methods based on optimization were developed such as the moving horizon estimation, which are powerful tools for nonlinear systems. ${ }^{8}$ The moving horizon estimation is a method based on online optimization using available information (e.g., data, knowledge about the process, operating conditions) as constraints and cost function for relating past measurements and the process model, all over a moving observation horizon. The aim of having a moving observation horizon is to include new measurements and eliminate the old ones in this horizon as time goes on. ${ }^{9}$ The applications of this estimation method are extended along many fields of engineering, such as the systems with delayed measurements, parameters estimation, uncertain systems, among others. ${ }^{8,10,11}$

The main contribution of this paper consists in the design of a nonlinear observer for the online estimation of slurry properties based on a phenomenological based semi-physical model (PBSM) describing the main phenomena of the system. The proposed observer approach is based on moving horizon estimation considering the original structure of model and each one of the terms from the nonlinear mathematical model. This type of processes can be generalized to any multiphase mixture moving through pipes assemblies in a process plant. In addition, and taking into account the inherent disturbances that can affect the mentioned transport process, and the difficulty to characterize this kind of fluids, one approximation for the disturbances or unknown inputs (UIs) is considered into the design, producing the proposed nonlinear moving horizon estimator (NLMHE). Thereby, based on the process knowledge, a feasible region for the UIs (or disturbances) is established, and from this, the UIs are approximated as random values into that region. Consequently, one NLMHE, without restrictions in the model structure and considering the UIs, is presented. 
The remainder of this paper is organized as follows. In Section 2, the estimation problem and the process model for transporting a mineral slurry are exposed. In Section 3, the necessary mathematical tools for the design of the NLMHE are introduced. Next, the proposed estimator, the simulation results, and the discussions related to these results, including the online estimation of both the density and viscosity of a mineral slurry flowing through a pipe-and-fittings assembly (PFA), are presented in Section 4. Finally, the main conclusions and some ideas for further research are drawn in Section 5.

\section{Notation}

In this work, column vectors are denoted in lower case and bold style (e.g., $\mathbf{x}$ ), while the matrices are written in upper case and bold style (e.g., M). Real numbers are denoted by $\mathbb{R}$, all the non-negative real numbers by $\mathbb{R}_{\geq 0}$, and the positive integer numbers by $\mathbb{Z}_{\geq 0}$. Finally, the dependency in continuous time and discrete time are denoted by $(t)$ and $(k)$, respectively.

\section{Transport process of a mineral slurry}

For transporting a mineral slurry through a PFA, the existence of a differential pressure between the inlet and outlet points of the pipeline is required. This differential pressure indicates the required energy to overcome the friction forces that are generated between fluid layers and fluid and pipe surface. However, the flow through the PFA is conditioned by the fluid properties (such as density and viscosity), which are strongly related to laminar

or turbulent flow regime, characterized by the Reynolds number. ${ }^{12}$ This number directly affects the calculations of friction factor, energy losses and pressure drop of a fluid flowing through a PFA.

During the process of a mineral slurry (Figure 1), the solids (minerals) are normally mixed with water to obtain a slurry, which can be pumped through different process units. 
This kind of fluids can exhibit non-Newtonian behavior due to the presence of phases in the mixture and, as a result, modify the mixture properties (density and viscosity). ${ }^{12}$ Taking into account that the Reynolds number depends on the density and viscosity, this and the flow behavior of the fluid (as a Newtonian or non-Newtonian fluid) impose conditions to the slurry transport through the PFA. Therefore, the non-Newtonian behaviors must be characterized in order to obtain a correct estimation of the slurry properties. These properties are commonly estimated since, in many cases, there are no available devices for measuring them and, when available, their use might be quite expensive. ${ }^{13,14}$

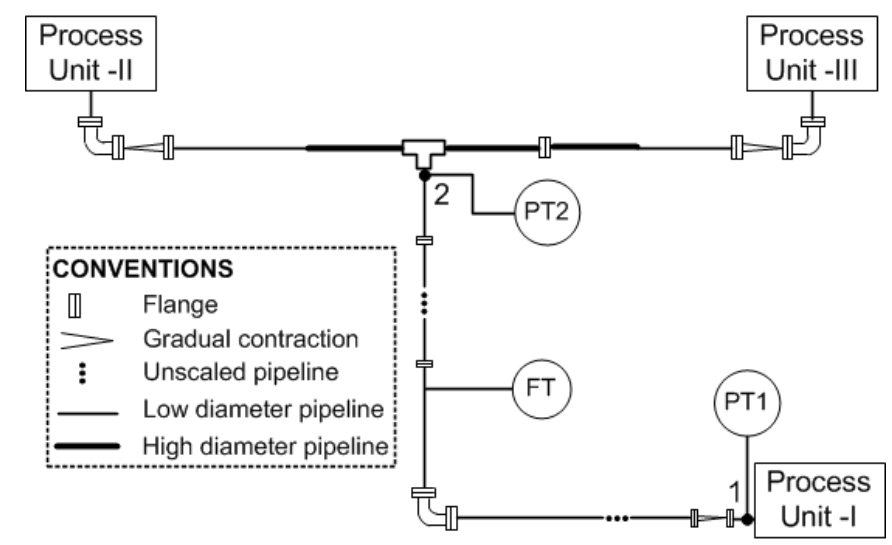

Figure 1: Basic scheme for the transport process of a pulp.

In these processes, the solids concentration in the mixture cannot be known with certainty since features and supply conditions of the raw material constantly change. Therefore, this concentration is often considered as an unknown input in the transport process. Nevertheless, the online estimation of both density and viscosity of a mineral slurry has often been approached using state observers based on empirical models and neural networks, and without considering that solids concentration constantly changes. ${ }^{4,15}$ Nonetheless, some works like the one proposed in Ref. 16 have tackled the estimator design based on phenomenological models. In the mentioned work, an unknown-input observer based on a PBSM is proposed, for estimating both density and viscosity of a mineral slurry. The relevance of this observer is that considers a PBSM that allows separating the 
estimation of the density and viscosity based on the conservation principles of mass and momentum, the main phenomena that govern the slurry flow and its rheological behavior. However, the linear observer proposed there, limits the observer performance to a region near the point used to linearize the model. Removing this restriction is the aim of this paper.

In the case of non-linear observers, there exist many options that establish a set of restrictions or conditions for mathematical forms of nonlinearities in the model equations. These options establish different model structures and transformations of the model equations to propose an observer structure. The main difficulty of these approaches is that they are quite restrictive regarding the model structure since were developed for a specific class of system or process. ${ }^{1}$ In the transport process of a mineral slurry flowing through a PFA, the main nonlinearities can be found in the terms for calculating the energy losses and the friction factor. Thus, the process model used for this development, proposed in Ref. 16 , is briefly presented below.

\subsection{Flow model of a slurry}

From mass and energy conservation principles, a PBSM (see Ref. 17) for the transport of a mineral slurry can be represented by the following balance equations: ${ }^{16}$

$$
\begin{aligned}
\frac{d \rho_{2}(t)}{d t}= & {\left[\rho_{1}(t)-\rho_{2}(t)\right] \frac{Q(t)}{V}, } \\
\frac{d Q(t)}{d t}= & \frac{A}{L}\left[-\frac{P_{2}(t)}{\rho_{2}(t)}+\frac{\dot{m}_{1}(t)}{\dot{m}_{2}(t)} \frac{P_{1}(t)}{\rho_{1}(t)}-g z_{2}+\frac{\dot{m}_{1}(t)}{\dot{m}_{2}(t)} g z_{1}\right] \\
& +\left[-\frac{\dot{m}_{1}(t)}{\dot{m}_{2}(t)} h_{f_{1 \rightarrow H P}}(t)-h_{f_{H P \rightarrow 2}}(t)\right]
\end{aligned}
$$

where $t \in \mathbb{R}_{\geq 0}$, and the subindices 1,2 and HP are the inlet, outlet and homogenization point, respectively. In (1), $\rho_{1}\left[\frac{\mathrm{kg}}{\mathrm{m}^{3}}\right]$ and $\rho_{2}\left[\frac{\mathrm{kg}}{\mathrm{m}^{3}}\right]$ are the inlet and outlet density, respectively, while $Q\left[\frac{\mathrm{m}^{3}}{\mathrm{~s}}\right], V\left[\mathrm{~m}^{3}\right], \mathrm{A}\left[\mathrm{m}^{2}\right]$ and $L[\mathrm{~m}]$ are the volumetric flow, the pipeline volume, the pipe cross area and length between the inlet and outlet points, respectively. Equations in (1) 
correspond to the mass and mechanical energy balance, respectively. These balances have been obtained considering disturbances in the inlet solids concentration $\left(w_{S, 1}\right)$ through its relationship with the inlet slurry density $\left(\rho_{1}\right)$. In addition, in order to consider that the outlet density and density inside the pipe are the same, i.e., with the same properties, the perfect agitation principle was assumed for the slurry inside the PFA $\left(\rho=\rho_{2}\right)$.

Equation (1b) represents the changes in the kinetic energy of the fluid produced by mechanical terms such as the flow, gravitational, and friction works acting on the fluid. In this case, the driving-machine work is not considered because no device is installed into the PFA. In (1b), $g\left[\frac{\mathrm{m}}{\mathrm{s}^{2}}\right]$ is the gravity, $P_{1}[\mathrm{~Pa}], P_{2}[\mathrm{~Pa}]$ are the inlet and outlet pressures, respectively, $z_{1}[\mathrm{~m}], z_{2}[\mathrm{~m}]$ are the height at inlet and outlet respect to a reference, respectively, $\dot{m}_{1}\left[\frac{\mathrm{kg}}{\mathrm{s}}\right]$ and $\dot{m}_{2}\left[\frac{\mathrm{kg}}{\mathrm{s}}\right]$ are the inlet and outlet slurry mass flow, respectively, and $h_{f_{1 \rightarrow H P}}\left[\frac{\mathrm{m}^{2}}{\mathrm{~s}^{2}}\right]$ and $h_{f_{H P \rightarrow 2}}\left[\frac{\mathrm{m}^{2}}{\mathrm{~s}^{2}}\right]$ are the friction losses between the inlet and homogenization points, and this latter and the outlet point in the PFA. The homogenization point (HP) corresponds to that point in the PFA in which the slurry is homogenized after a disturbance takes place.

Since the slurry is considered as a mixture of solids with water, the relationship between the phases concentration and the slurry density is given by the known harmonic weighted average

$$
\rho(t)=\frac{1}{\frac{w_{W}(t)}{\rho_{W}}+\frac{w_{S}(t)}{\rho_{S}}},
$$

being $w_{W}$ and $w_{S}$ the water and solids concentrations, respectively, and $\rho_{W}$ and $\rho_{S}$ the water and average solid densities, respectively. It should be mentioned that the subscript $i$ is used to indicate the inlet or outlet points of the slurry in the PFA ( $i=1$ for inlet and $i=2$ for the outlet). From (2), it is possible to obtain the solids concentration $w_{S, 2}$ after $\rho_{2}$ 
is estimated. The set of constitutive equations used to compute $h_{f_{1 \rightarrow 2}}$ is the following:

$$
\begin{aligned}
h_{f_{1 \rightarrow 2}}(t) & =\left(K_{p}(t)+\Sigma K_{F}(t)\right) \frac{1}{2} \frac{Q^{2}(t)}{A^{2}}, \\
K_{p}(t) & =f_{D_{i}}(t)\left(\frac{D}{L}\right), \\
K_{F}(t) & =\frac{K_{1}}{N_{R e_{i}}(t)}+K_{\infty}\left(1+\frac{1}{I D}\right), \\
f_{D_{i}}(t) & =\left\{-2 \log \left[\frac{\epsilon / D}{3.1}-\frac{5.02}{N_{R e_{i}}(t)} \log \left(\frac{\epsilon / D}{3.71}+\frac{14.5}{N_{R e_{i}}(t)}\right)\right]\right\}^{-2}, \\
N_{R e_{i}}(t) & =\frac{v_{i}(t) D \rho_{i}(t)}{\mu_{i}(t)} .
\end{aligned}
$$

Equation (3a) is used to compute the energy losses in the pipeline and the fittings, whereas $K_{p}$ and $K_{F}$ in (3b) and (3c) are the $K$-factors of losses for pipe and fittings, respectively. The $K$-factor calculation for the fittings is done based on the type of element and its union to the pipe, for instance, welded, flanged, threaded, etc. These features are considered into the parameters $K_{1}$ and $K_{\infty}$, which are taken from Hooper in 1981. In (3c), ID corresponds to the pipe inner diameter in inches.

The friction factor is calculated using (3d) (the Shacham Equation Ref. 19), which includes highly nonlinear terms. In (3d), $\epsilon$ is the absolute pipe roughness while the Reynolds number $\left(N_{R e_{i}}\right)$ is computed by (3e), being $\mu_{i}[\mathrm{~Pa} \mathrm{~s}]$ the slurry viscosity. In Ref. 16 , the proposed observer assumes the density as a state variable (with differential equation) while the viscosity is estimated using an algebraic expression, which considers the operating conditions for the flow in the PFA and the rheological behavior of pseudoplastic fluids. This latter behavior is a feature of this kind of slurries. Then, taking into account the rheological behavior and flow conditions through a pipeline, the viscosity expression is given by

$$
\mu(t)=K_{\mu}(t)\left[\left(\frac{3 n(t)+1}{4 n(t)}\right) \frac{8 \bar{u}(t)}{D_{T}}\right]^{n(t)-1},
$$

where $\mu, K_{\mu}$ and $n$ are the viscosity, the consistency factor and the behavior index, respec- 
tively. Moreover, $\bar{u}[\mathrm{~m} / \mathrm{s}]$ and $D_{T}[\mathrm{~m}]$ are the average velocity in the line and the diameter of pipe, respectively. The factor in brackets in (4) corresponds to the shear rate $(\dot{\gamma})$ for non-Newtonian fluids in a pipe. ${ }^{20}$ The detailed deduction of the viscosity expression and, the explanation on how to compute the parameters $K_{\mu}$ and $n$ are presented in Appendix.

The expressions (1) - (4) form the equations of the PBSM, from which the proposal presented in this paper is developed. Based on these equations, the model nonlinearities can be noticed, such as the logarithm function in (3d), which involves the properties to be estimated (density and viscosity). Due to the nature of these equations (obtained from different conservation principles) and the resulting structure, the nonlinear state transformation-based techniques proposed for the design of nonlinear observers (see e.g., Ref. 21-23) are not useful tools for the transport model since it is not possible to apply any useful mathematical transformation. ${ }^{1}$

\section{Nonlinear moving horizon estimator with bounded un- known input}

In order to overcome the estimation problem for nonlinear systems with unknown inputs, a NLMHE is designed. This estimator can be used to deal with nonlinear systems that do not comply with the structural conditions in the model equations, or which do not satisfy the restrictions for the nonlinear terms proposed in the literature for the design of nonlinear estimators. Besides, the proposed estimator considers an approximation by intervals for the dynamics of the UIs.

The moving horizon approach is considered for developing an estimator of the state variables, which describes the system condition at time $t$ based on the difference between the values of the measurement and estimation for the measured variables from the process. However, since the NLMHE needs to know all variables and parameters that will not be estimated, one method for modeling the dynamics of the UIs is required. From this, and 
when the UIs cannot be modeled but can be bounded, these are assumed as random inputs among their bounding values. In addition, the interval-observer approach is used in order to determine and to evaluate the operating region for the estimator.

The proposed NLMHE estimates state variables based on the solution of an optimization problem constrained to the process model, and using a random value for the UIs into its feasible region. Thus, the interval approach will be developed by solving the proposed NLMHE both for the admissible minimum and maximum value of the UI, with the aim determining the feasible region for estimation of the state variables. Thereby, the admissible minimum and maximum value of the UI can be established from the knowledge of the operating range for solids concentration, which is related to the slurry density as it is evident from the known harmonic weighted average (see (2)). The main tools used in the development of this proposal are presented in Sections 3.1 and 3.2.

\subsection{Moving horizon state estimator}

According to Boegli in 2014, the moving horizon estimation is an optimal control approach pointing to find the system states, which are more consistent with current and past inputoutput data and the available process model. The aim of this method is to estimate the current states by solving a least squares optimization problem constrained to the model process, and considering a fixed horizon of measurements $\left\{y_{0}, y_{1}, \ldots, y_{T-1}\right\}$, which penalizes the deviation between the measurements and estimated outputs. ${ }^{25,26}$ Thus, the estimation problem is formulated over a moving horizon of fixed length, which is moved in the time and at each time step that horizon moves to the next data, the past measurement is discarded and a new measurement is taken into account. Then, since the moving horizon is changing at each time instant, a new optimization problem is solved and the estimation is obtained. ${ }^{26,27}$

In order to state the observer design, consider a continuous-time system model of the 
form

$$
\begin{aligned}
\frac{d \mathbf{x}(t)}{d t} & =\mathbf{f}_{\mathbf{c}}(\mathbf{x}(t), \mathbf{u}(t), \mathbf{d}(t))+\boldsymbol{\omega}(t), \\
\mathbf{y}(t) & =\mathbf{g}_{\mathbf{c}}(\mathbf{x}(t))+\boldsymbol{v}(t),
\end{aligned}
$$

where $\mathbf{x} \in \mathcal{X} \subseteq \mathbb{R}^{n_{x}}, \mathbf{u} \in \mathcal{U} \subseteq \mathbb{R}^{n_{u}}, \mathbf{d} \in \mathcal{D} \subseteq \mathbb{R}^{n_{d}}$ and $\mathbf{y} \in \mathcal{Y} \subseteq \mathbb{R}^{n_{y}}$ are the state, manipulated input, unknown input and output vector, respectively, while $\omega \in \mathcal{W} \subseteq \mathbb{R}^{n_{x}}$ and $v \in \mathcal{V} \subseteq \mathbb{R}^{n_{y}}$ are the uncertainties vectors in the model and measurements, respectively. Moreover, $\mathbf{f}_{\mathbf{c}}: \mathbb{R}^{n_{x}} \times \mathbb{R}^{n_{u}} \times \mathbb{R}^{n_{d}} \rightarrow \mathbb{R}^{n_{x}}$ and $\mathbf{g}_{\mathbf{c}}: \mathbb{R}^{n_{x}} \rightarrow \mathbb{R}^{n_{y}}$ are the nonlinear mapping functions for the dynamics and output equations, respectively. In general, to perform any computation, the continuous-time version of the process model must be discretized. Thus, based on the finite difference discretization scheme and Taylor series expansion, the discrete-time version of the model in (5) is obtained as

$$
\begin{aligned}
\mathbf{x}(k+1) & =\mathbf{f}(\mathbf{x}(k), \mathbf{u}(k), \mathbf{d}(k))+\boldsymbol{\omega}(k), \\
\mathbf{y}(k) & =\mathbf{g}(\mathbf{x}(k))+\boldsymbol{v}(k),
\end{aligned}
$$

being $k \in \mathbb{Z}_{\geq 0}$ the discrete-time index, and related to the continuous time by $t=k \Delta t$, for $\Delta t$ the sampling time (or discretization period).

It should be noted that for the case of stiff systems, the system discretization is more complex, and techniques such as single shooting, collocation, etc, are required for nonlinear systems. ${ }^{28}$ Based on sensors in the real process, the sampling time is established and the discrete versions of the known inputs are the observations from these sensors. Thus, for the unknown inputs, the discrete approximation is obtained selecting one random value for each UI among the bounded values for these inputs at each time instant. Hence, 


$$
\begin{aligned}
& \mathcal{X}=\left\{\mathbf{x} \in \mathbb{R}^{n_{x}} \mid \mathbf{x}(k) \in[\underline{\mathbf{x}}, \overline{\mathbf{x}}], \forall k\right\}, \\
& \mathcal{U}=\left\{\mathbf{u} \in \mathbb{R}^{n_{u}} \mid \mathbf{u}(k) \in[\underline{\mathbf{u}}, \overline{\mathbf{u}}], \forall k\right\}, \\
& \mathcal{D}=\left\{\mathbf{d} \in \mathbb{R}^{n_{d}} \mid \mathbf{d}(k) \in[\underline{\mathbf{d}}, \overline{\mathbf{d}}], \forall k\right\}, \\
& \mathcal{Y}=\left\{\mathbf{y} \in \mathbb{R}^{n_{y}} \mid \mathbf{y}(k) \in[\underline{\mathbf{y}}, \overline{\mathbf{y}}], \forall k\right\},
\end{aligned}
$$

where $\underline{\mathbf{x}}$ and $\overline{\mathbf{x}}$ are the vectors of minimum and maximum values of state variables, respectively. In the same way, this notation is valid for other variables, i.e., $\mathbf{u}, \mathbf{d}$ and $\mathbf{y}$.

Based on the discrete-time version of the model in (6), and keeping in mind that the moving horizon estimation solves a constrained optimization problem over a finite horizon, the estimator at current time $T$ is designed based on the following problem formulation: ${ }^{24}$

$$
\min _{\tilde{\mathbf{x}}, \tilde{\boldsymbol{\omega}}} \phi_{T}(\tilde{\mathbf{x}}, \tilde{\boldsymbol{\omega}})
$$

subject to

$$
\begin{aligned}
\mathbf{x}(k+1) & =\mathbf{f}(\mathbf{x}(k), \mathbf{u}(k), \mathbf{d}(k))+\boldsymbol{\omega}(k), \\
\mathbf{y}(k) & =\mathbf{g}(\mathbf{x}(k))+\boldsymbol{v}(k),
\end{aligned}
$$

for all $k \in[T-N, T]$, and being $\tilde{\mathbf{x}}=\left\{\tilde{\mathbf{x}}_{1}, \tilde{\mathbf{x}}_{2}, \cdots, \tilde{\mathbf{x}}_{T-1}, \tilde{\mathbf{x}}_{T}\right\}$ and $\tilde{\boldsymbol{\omega}}=\left\{\tilde{\omega}_{1}, \tilde{\omega}_{2}, \cdots, \tilde{\omega}_{T-1}, \tilde{\omega}_{T}\right\}$ the sequences to be found along the moving horizon until $t=T$ obtained from the solution of (8a), whereas $N \in \mathbb{Z}_{>0}$ is the length of the moving horizon. On the other hand, the cost function in (8a) can be expressed as

$$
\phi_{T}(\tilde{\mathbf{x}}, \tilde{\boldsymbol{\omega}})=\left\|\tilde{\mathbf{x}}_{T-N}-\overline{\mathbf{x}}_{T-N}\right\|_{\mathbf{P}_{T-N}^{-1}}^{2}+\sum_{k=T-N+1}^{T}\|\mathbf{y}(k)-\tilde{\mathbf{y}}(k)\|_{\mathbf{R}_{k}^{-1}}^{2}+\|\tilde{\boldsymbol{\omega}}(k)\|_{\mathbf{Q}_{k}^{-1}}^{2},
$$


where $\phi_{T}: \mathbb{R}^{n_{x}} \times \mathbb{R}^{n_{y}} \times \mathbb{R}^{n_{x}} \rightarrow \mathbb{R}$, the first term in the right side of (9) is the arrival cost that summarizes past information between $t_{0}$ and $t_{T-N}, \mathbf{y}$ and $\tilde{\mathbf{y}}$ are the vectors of the measurements and optimized outputs (measured state variables), respectively, while $\mathbf{Q}_{\mathbf{k}}^{-1}, \mathbf{R}_{\mathbf{k}}^{-1}$ and $\mathbf{P}_{\mathbf{T}-\mathbf{N}}^{-1}$ are the inverses of the weighting matrices regarding model uncertainty, measurement uncertainty and covariance of all available past information, respectively. The couple $\left(\overline{\mathbf{x}}_{T-N}, \mathbf{P}_{\mathbf{T}-\mathbf{N}}\right)$ can be considered as a regularization term for the first estimate, namely $\hat{\mathbf{x}}_{T-N}$, along $N$, and which ensures the stability of the proposed scheme mainly for short horizons. ${ }^{24}$ There are different ways to compute $\overline{\mathbf{x}}_{T-N}$ and $\mathbf{P}_{\mathbf{T}-\mathbf{N}}$ depending on the nature of the system (linear or nonlinear). For example, the nonlinear filters are commonly used for estimating the state variables and covariance matrix at $T-N$. These filters obtain the required values in the arrival cost function $\left(\overline{\mathbf{x}}_{T-N}, \mathbf{P}_{\mathbf{T}-\mathbf{N}}\right)$ from data history about the previous evolution of the system up to the first value of the current estimation horizon considered. For nonlinear systems, the common methods are the Extended Kalman filter (EKF), the unscented Kalman filter (UKF) and the particle filter (PF). ${ }^{24,26,28}$

In addition, if the input vector $\mathbf{u}$ is considered as independent input without uncertainty and the model uncertainty $\tilde{\boldsymbol{\omega}}$ is not considered, the last term in (9) can be dropped, ${ }^{24}$ and then (9) is reduced to

$$
\phi_{T}(\tilde{\mathbf{x}})=\left\|\tilde{\mathbf{x}}_{T-N}-\overline{\mathbf{x}}_{T-N}\right\|_{\mathbf{P}_{T-N}^{-1}}^{2}+\sum_{k=T-N+1}^{T}\|\mathbf{y}(k)-\tilde{\mathbf{y}}(k)\|_{\mathbf{R}_{k}^{-1}}^{2} .
$$

Then, determining the regularization pair $\left(\overline{\mathbf{x}}_{T-N}, \mathbf{P}_{\mathbf{T}-\mathbf{N}}\right)$ and solving the optimization problem in (10), vector $\tilde{\mathbf{x}}$ is obtained, which corresponds to the estimated states $(\hat{\mathbf{x}})$. However, although the moving horizon estimation method allows to obtain the estimation of the state variables, this method needs to know all variables and parameters that will not be optimized. Hence, it is necessary to establish a way to include the UI into the estimation problem. The proposal of this paper for considering the UI is presented in Section 3.2. 


\subsection{Approximation of the unknown inputs}

Assuming that the UI can be bounded from the knowledge about the process, this input is taken as a random value between the lower and upper values of its admissible domain at each time instant $k$. From this, two different NLMHE are designed, one for each bound of the UI, and thus obtaining the feasible region for the state estimation.

If the UI can be restricted to the admissible region defined by the range $[\underline{\mathbf{d}}, \overline{\mathbf{d}}]$, the state estimation for one random value of $\mathbf{d}^{r}(k)$ will be found between the estimated states for $\underline{\mathbf{d}}$ and $\overline{\mathbf{d}}$. Considering the proposal for the NLMHE, the optimization problem when $\mathbf{d}=\overline{\mathbf{d}}$ is stated as follows:

$$
\min _{\tilde{\mathbf{x}}}\left\|\tilde{\mathbf{x}}_{T-N}-\overline{\mathbf{x}}_{T-N}\right\|_{\mathbf{P}_{T-N}^{-1}}^{2}+\sum_{k=T-N+1}^{T}\|\mathbf{y}(k)-\tilde{\mathbf{y}}(k)\|_{\mathbf{R}_{k}^{-1}}^{2}
$$

subject to

$$
\begin{aligned}
& \mathbf{x}(k+1)=f(\mathbf{x}(k), \mathbf{u}(k), \overline{\mathbf{d}}(k)), \\
& \mathbf{y}(k)=g(\mathbf{x}(k)),
\end{aligned}
$$

for all $k \in[T-N, T]$. In (11a), $\overline{\mathbf{x}}$ corresponds to the solution of the optimization problem in (11) when the disturbance takes the value $\overline{\mathbf{d}}$, and the last value of this sequence is the state estimation at time $T\left(\overline{\hat{\mathbf{x}}}_{T \mid T-N}\right)$. On the other hand, considering $\underline{\mathbf{d}}$, the optimization problem is stated as follows:

$$
\min _{\underline{\underline{x}}}\left\|\tilde{\mathbf{x}}_{T-N}-\overline{\mathbf{x}}_{T-N}\right\|_{\mathbf{P}_{T-N}^{-1}}^{2}+\sum_{k=T-N+1}^{T}\|\mathbf{y}(k)-\tilde{\mathbf{y}}(k)\|_{\mathbf{R}_{k}^{-1}}^{2}
$$


subject to

$$
\begin{aligned}
& \mathbf{x}(k+1)=f(\mathbf{x}(k), \mathbf{u}(k), \underline{\mathbf{d}}(k)), \\
& \mathbf{y}(k)=g(\mathbf{x}(k)),
\end{aligned}
$$

for all $k \in[T-N, T]$, being $\underline{\tilde{x}}$ the solution of the optimization problem (12) and $\underline{\hat{x}}_{T \mid T-N}$ the optimal state estimation when disturbances take the value $\underline{\mathbf{d}}$. Although (11a) and (12a) have the same structure, they are different in the $\tilde{\mathbf{y}}(k)$ value obtained from the model

equations using either $\overline{\mathbf{d}}$ or $\underline{\mathbf{d}}$, respectively. Consequently, the solution $\hat{\mathbf{x}}_{T \mid T-N}^{*}$, for an random value $\mathbf{d}^{r}(k)$, should hold

$$
\hat{\mathbf{x}}_{T \mid T-N} \leq \hat{\mathbf{x}}_{T \mid T-N}^{*} \leq \overline{\hat{\mathbf{x}}}_{T \mid T-N}
$$

Then, without considering dynamics for the UI and using the interval-observer approach, it is possible to determine the feasible region of estimation. In addition, the proposed estimator is not restricted to a stationary state because the nonlinearities of the model are considered, and the UI is bounded and approximated as a random value. Algorithm 1 collects the required steps to follow for designing the proposed NLMHE.

\section{Simulation results}

According to Section 3, and since the only available measurement from the process is $Q$ and the pressure drop $\left(\Delta P=P_{2}-P_{1}[\mathrm{~Pa}]\right)$, the optimization problem for the design of the NLMHE for slurry properties can be written as follows: 


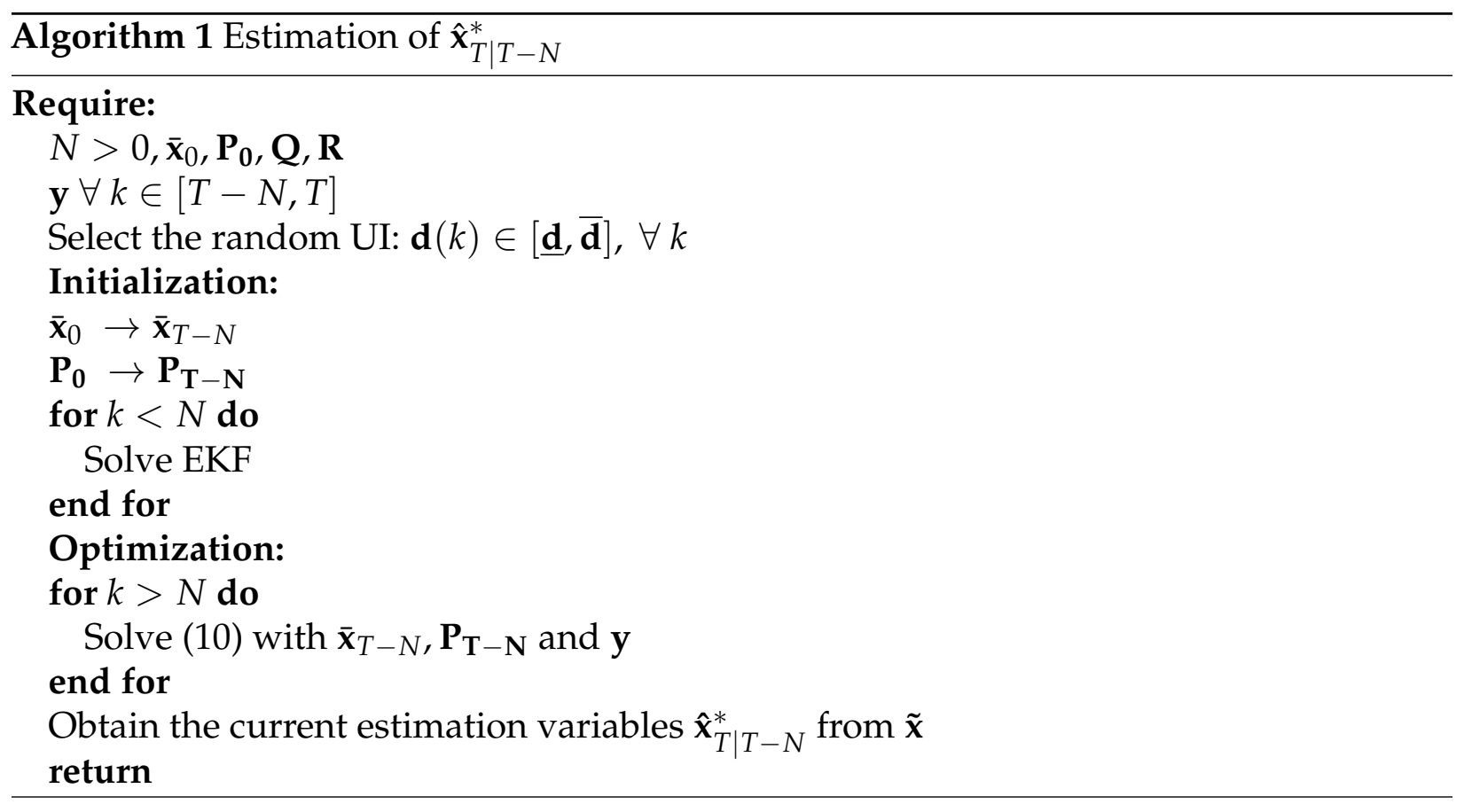

$$
\min _{\tilde{\mathbf{x}}}\left\|\tilde{\mathbf{x}}_{T-N}-\overline{\mathbf{x}}_{T-N}\right\|_{\mathbf{P}_{T-N}^{-1}}^{2}+\sum_{k=T-N+1}^{T}\left\|Q_{m}(k)-\tilde{Q}(k)\right\|_{\mathbf{R}_{k}^{-1},}^{2}
$$

subject to

$$
\begin{aligned}
& \rho_{2}(k+1)=f_{1}(\mathbf{x}(k), \mathbf{u}(k), \mathbf{d}(k)), \\
& Q(k+1)=f_{2}(\mathbf{x}(k), \mathbf{u}(k), \mathbf{d}(k)), \\
& \mathbf{y}(k)=\tilde{Q},
\end{aligned}
$$

for all $k \in[T-N, T]$, where $Q_{m}$ and $\tilde{Q}$ are the measured outlet and the optimized outlet, respectively, while the estimated states $\hat{\rho_{2}}$ and $\hat{Q}$ are obtained from $\tilde{\mathbf{x}}$ and the process model in $(14 \mathrm{~b})$ - (14d). In (14), $f_{1}$ and $f_{2}$ correspond to the discrete versions of the model expressions (1a) and (1b), respectively. According to Algorithm 1, the solution to the optimization problem (14) was obtained in two stages: the former uses the EKF to determine the terms in the arrival cost function based on the past information to the 
Table 1: Classification of the process variables.

\begin{tabular}{|l|l|}
\hline Type & Symbol \\
\hline State variables & $\mathbf{x}=\left[\rho_{2}, Q\right]^{T}$ \\
\hline Known inputs & $\mathbf{u}=\left[P_{1}, P_{2}, \mu_{2}\right]^{T}$ \\
\hline Unknown inputs & $\mathbf{d}=w_{S, 1}$ \\
\hline Measured outlets & $\mathbf{y}=Q$ \\
\hline
\end{tabular}

estimation horizon, and considering $\mathbf{P}_{\mathbf{T}-\mathbf{N}}=1$ and $\mathbf{R}_{\mathbf{k}}=0.0001$ since the unique available measure in the process is the volumetric flow, which is obtained from the flowmeter with a measuring uncertainty of $0.0001 \mathrm{~m}^{3} / \mathrm{s}$. In the latter, the results of the first stage for $T-N$ are replaced in (14a) to solve the optimization problem and determining the estimated values. From the proposed estimator and the model presented in Section 2, the simulation results for the online estimation of the density and viscosity of a mineral slurry flowing through a PFA are presented in this section. These results were obtained based on the classification of process variables shown in Table 1, and the model parameters presented in Table 2.

It should be noted that although $\mu_{2}$ is one of the variables to estimate, it is considered as a known value in this paper since the estimation structure does not consider the viscosity as state variable but is estimated from algebraic equations, which are in function of the states. Therefore, the initial iteration is done considering a seed value, which is replaced by the estimated value in the next iteration since the slurry viscosity is computed after the state variables $\left(Q\right.$ and $\left.\rho_{2}\right)$ are estimated.

All results presented in this paper were obtained using the software package NPSOL of

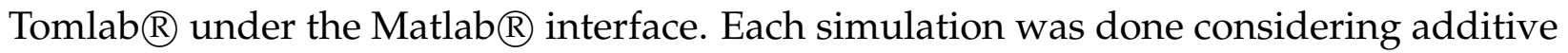
noise in the measured variable (volumetric flow, $Q$ ). In addition, two disturbances in the solids initial concentration were applied to evaluate the performance of the proposed NLMHE, and compared with the linear observer proposed in Ref. 16. 
Table 2: Simulation and model parameters.

\begin{tabular}{|l|c|c|c|}
\hline Parameter & Symbol & Value & Units \\
\hline Simulation time & $t_{s}$ & 100 & $\mathrm{~s}$ \\
\hline Sampling time & $\Delta t$ & 0.1 & $\mathrm{~s}$ \\
\hline Length of moving horizon & $N$ & 5 & - \\
\hline Initial time for estimation & $T_{0}$ & 10 & $\mathrm{~s}$ \\
\hline Tolerance for the optimization & $T_{0} l_{x}$ & $10^{-6}$ & - \\
\hline Pipe length & $L$ & 11.766 & $\mathrm{~m}$ \\
\hline Pipe cross area & $A$ & 0.0323 & $\mathrm{~m}^{2}$ \\
\hline Pipe volume & $V$ & 0.3798 & $\mathrm{~m}^{3}$ \\
\hline Solids density & $\rho_{S}$ & 2600.4 & $\frac{\mathrm{kg}^{3}}{\mathrm{~m}^{3}}$ \\
\hline Water density & $\rho_{W}$ & 1000 & $\frac{\mathrm{kg}^{3}}{\mathrm{~m}^{3}}$ \\
\hline Pipe roughness & $\epsilon$ & $1.5 \times 10^{-6}$ & $\mathrm{~m}$ \\
\hline Pipe diameter & $D$ & 0.2027 & $\mathrm{~m}$ \\
\hline Height of inlet point & $z_{1}$ & 0 & $\mathrm{~m}$ \\
\hline Height of outlet point & $z_{2}$ & 12.58 & $\mathrm{~m}$ \\
\hline
\end{tabular}

\subsection{Validation of the proposed NLMHE}

The performance of the proposed NLMHE was validated in simulation considering a real data set from a Colombian industry of mineral processing. ${ }^{16}$ Considered data set includes measurements of volumetric flow, pressures, and slurry density for a normal operating conditions of the process (without disturbances). Then, based on the available data set, the moving horizon was selected, and from this the NLMHE was designed. The validation results for the proposed estimator are presented in Figure 2. According to the results shown in this figure, it is possible to see that the proposed estimator follows the real behavior with small differences between the real flow and its estimation. Thus, the NLMHE estimates the state variables (measured and unmeasured), just using $Q$ into the cost function, with an estimation error lower that $2 \%$. In addition, the estimation is done considering additive noise in the measured variable and a random value for the inlet density (UI) within the considered range. The estimator was tested using a length of moving horizon $N=5$, which was selected as it is explained in Section 4.2.

It should be noted that since the real signal $Q_{m}$, with $100 \mathrm{~s}$ of duration and $\Delta t=0.1$ 

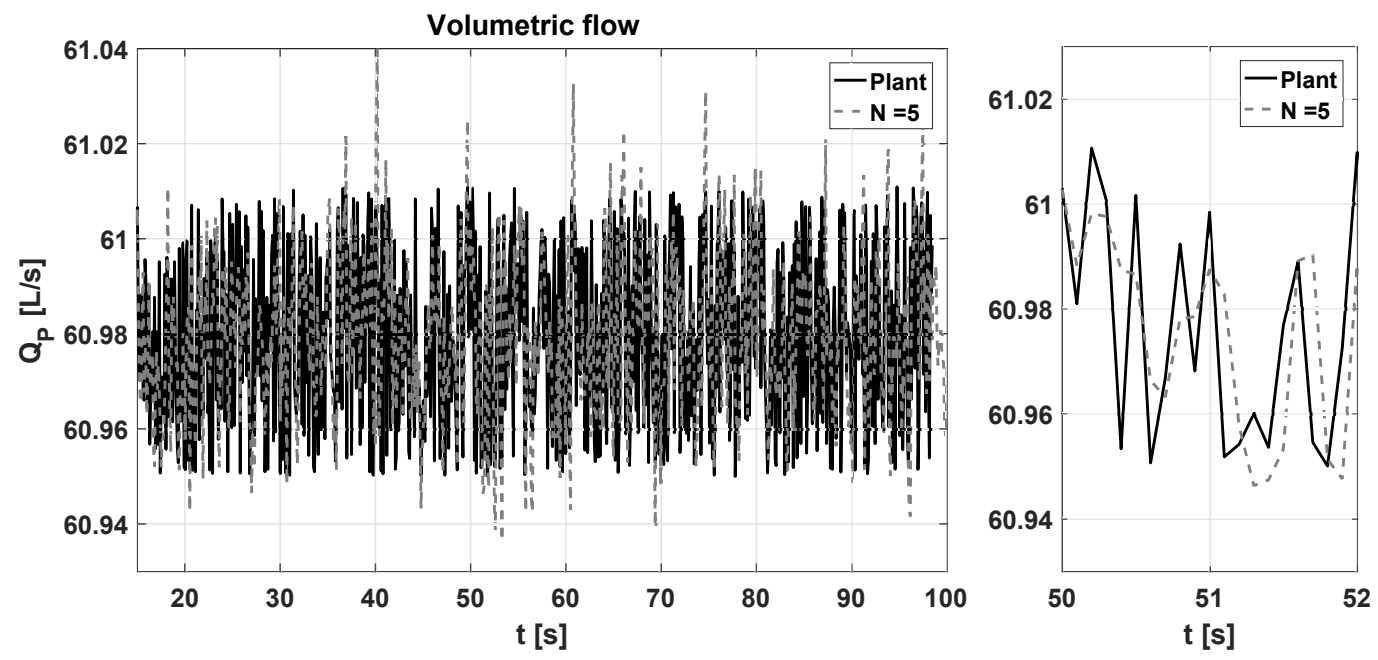

(a) Validation from real data set for the measured variable.
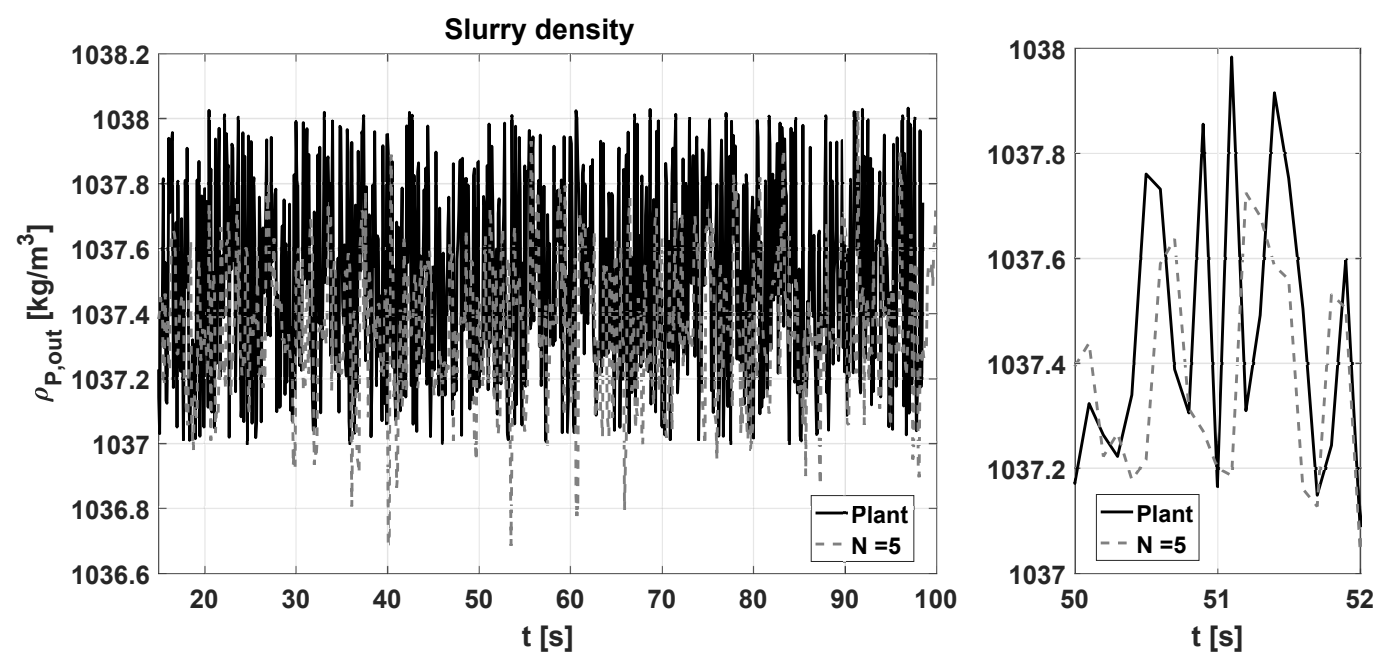

(b) Validation from real data set for the unmeasured variable.

Figure 2: Validation from real data set for the proposed NLMHE.

s, corresponds to a nominal operating point without disturbances, this data cannot be used to validate the observer performance when the disturbances take place. Therefore, to evaluate the performance of NLMHE considering disturbances in the solids concentration, the results presented hereafter were obtained from the operating point of process (for handling the real data) but introducing changes in the initial concentration of the solids. 


\subsection{Selecting the length of $N$}

Taking into account that the NLMHE requires a set of historical measurements, the length of $N$ must be selected. Therefore, based on the real data, a set of fifteen historical measurements is taken as the available data for establishing a suitable value of $N$. It is worth mentioning that each one of measurements and estimations obtained by the NLMHE has hereafter $\Delta t=0.1 \mathrm{~s}$ due to the restrictions for correct performance of the EKF. Thus, different sizes of $N$ were tested, and based on these results, the length of $N$ was fixed. The results above mentioned are shown in Figure 3. Once the estimation of the state variables is done, the slurry viscosity is computed by using these estimations and (4). The obtained result for this viscosity is presented in Figure 4.

The selection of $N$ was done based on the computing time per iteration $\left(t_{c, \text { iter }}\right)$, and the estimation error quantified using the Root Mean Square Error (RMS), the Residual Standard Deviation (RSD), and the Index of Agreement (IA). The corresponding equations for computing the above error indices are given by

$$
\begin{aligned}
& R M S=\sqrt{\frac{\sum_{i=1}^{n}\left(\mathbf{y}_{\mathbf{m}}-\hat{\mathbf{y}}\right)^{2}}{\sum_{i=1}^{n}\left(\mathbf{y}_{\mathbf{m}}\right)^{2}}}, \\
& R S D=\sqrt{\frac{\sum_{i=1}^{n}\left(\mathbf{y}_{\mathbf{m}}-\hat{\mathbf{y}}\right)^{2}}{n}}, \\
& I A=1-\frac{\sum_{i=1}^{n}\left(\mathbf{y}_{\mathbf{m}}-\hat{\mathbf{y}}\right)^{2}}{\sum_{i=1}^{n}\left(\mathbf{y}_{\mathbf{m}}^{\prime}-\hat{\mathbf{y}}^{\prime}\right)^{2}}
\end{aligned}
$$

where $\mathbf{y}_{\mathbf{m}}^{\prime}(i)=\mathbf{y}_{\mathbf{m}}(i)-\overline{\mathbf{y}}_{m}$ and $\mathbf{y}^{\prime}(i)=\mathbf{y}(i)-\overline{\mathbf{y}}_{m}$, and being $\overline{\mathbf{y}}_{m}$ the mean value of the measured variable. The RMS and RSD corresponding to the standard deviation of residual, and values close to zero indicate goodness-of-fit, while the IA indicates a suitable degree of fitting of the estimated and measured state variables for values above to 0.9. According to Table 3, each one of the evaluated error indices has similar values for the different 

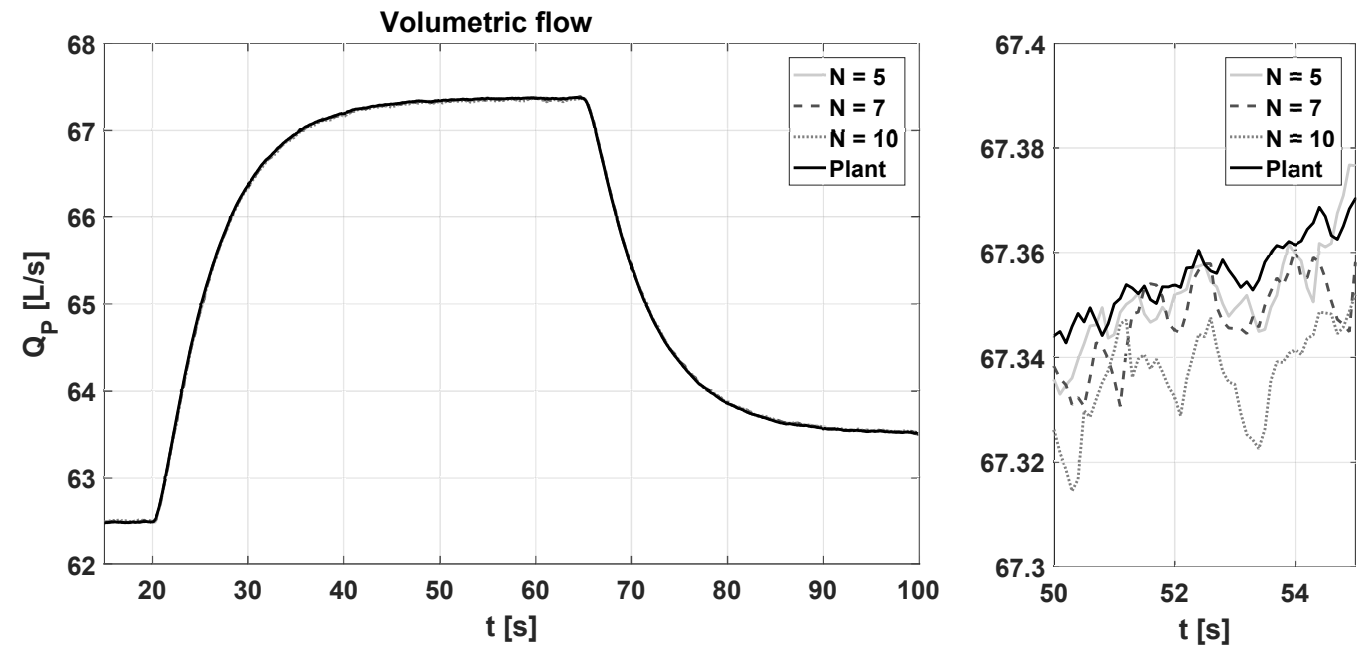

(a) Volumetric flow estimation for different values of $N$.
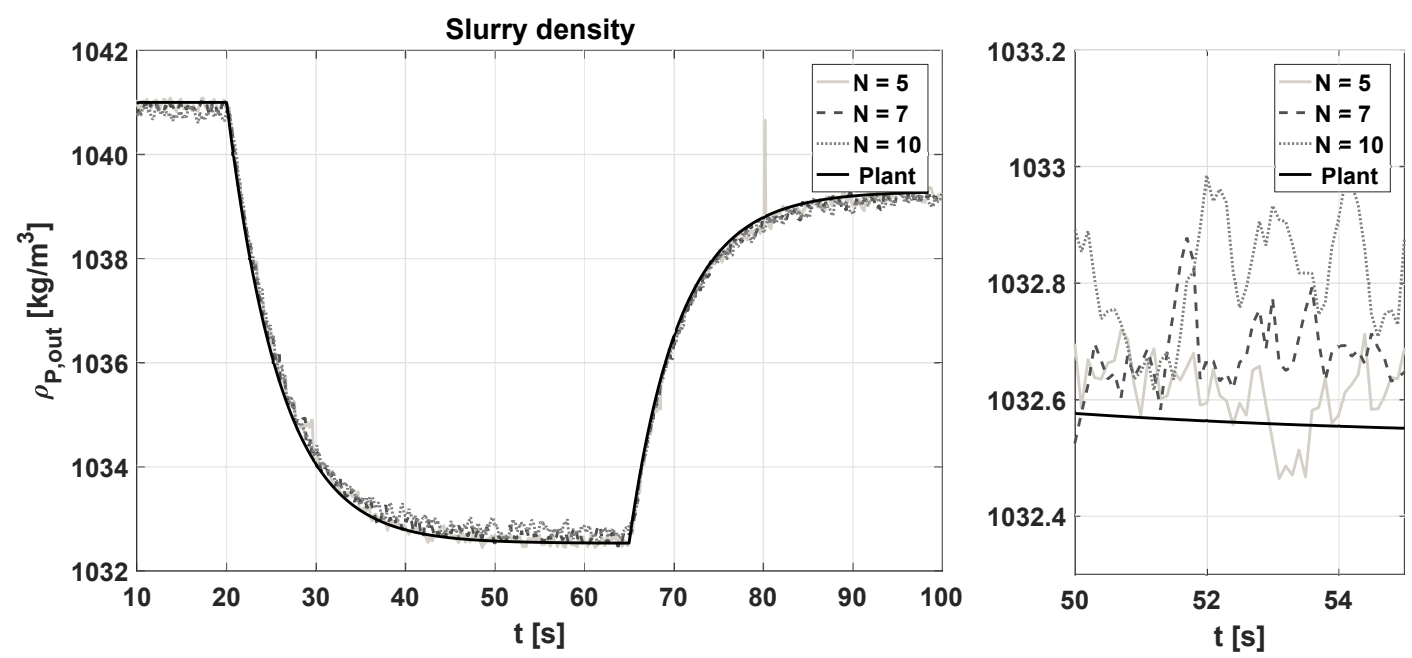

(b) Density estimation for different values of $N$.

Figure 3: Simulation of NLMHE for different values of $N$.

cases of $N$ tested, which allows to evidence that the proposed NLMHE follows the real trajectory of the state variables, and the estimation error is less than $2 \%$ in all the cases considered. However, regarding the computing time per iteration, which includes the stages to solve the EKF and the optimization problem in (14), it is significantly smaller for $N=5$ compared to the other cases tested. Therefore, based on the results in Table $3, N=5$ was selected for the simulations presented hereafter since that has the less computing time even for similar estimation errors to those obtained when $N=7$ and $N=10$.

It should be noted that most of the computing time is employed to solve the EKF stage 

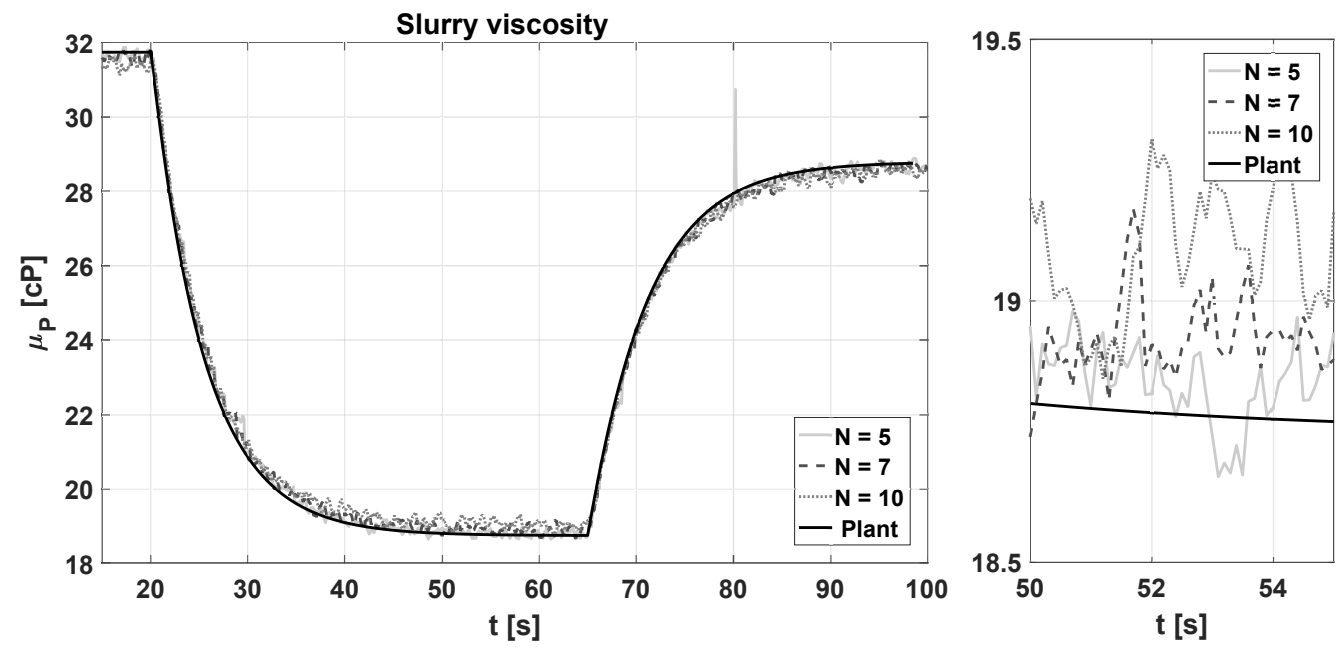

Figure 4: Viscosity estimation for different values of $N$.

with almost $75 \%$ of the total time. From this, more efficient alternatives than EKF for computing the arrival cost can be tested towards reducing the computing time.

\subsection{Approximation for the unknown inlet}

Taking into account that $N=5$, the proposed NLMHE was tested for the feasible interval of the UI. It should be recalled that the UI will be approximated as a random input between the bounds for the inlet density of slurry in the real process. Thereby, that value of the UI was obtained by using the command rand from Matlab $\mathbb{R}$. Since the transport of a mineral slurry involves operations with solids, $\underline{d}$ and $\bar{d}$ must consider the presence of those solids in the mixture. From the real data, the operating range for the slurry inlet density is

Table 3: Error indices and computational time for the state estimation and different values of $N$.

\begin{tabular}{|c|c|c|c|c|c|c|c|}
\hline \multirow{2}{*}{$\mathbf{N}$} & \multirow{2}{*}{$\mathbf{t}_{\mathbf{c}, \text { iter }}[\mathrm{s}]$} & \multicolumn{2}{|c|}{ RMS } & \multicolumn{2}{c|}{ RSD } & \multicolumn{2}{c|}{ IA } \\
\cline { 3 - 8 } & & $\hat{\mathbf{Q}}\left[\frac{\mathrm{L}}{\mathrm{s}}\right]$ & $\hat{\boldsymbol{\rho}}_{\mathbf{2}}\left[\frac{\mathrm{kg}}{\mathrm{m}^{3}}\right]$ & $\hat{\mathbf{Q}}\left[\frac{\mathrm{L}}{\mathrm{s}}\right]$ & $\hat{\boldsymbol{\rho}}_{\mathbf{2}}\left[\frac{\mathrm{kg}}{\mathrm{m}^{3}}\right]$ & $\hat{\mathbf{Q}}\left[\frac{\mathrm{L}}{\mathrm{s}}\right]$ & $\hat{\boldsymbol{\rho}}_{\mathbf{2}}\left[\frac{\mathrm{kg}}{\mathrm{m}^{3}}\right]$ \\
\hline $\mathbf{5}$ & 0.248 & $3.855 \times 10^{-5}$ & 0.005 & $9.826 \times 10^{-6}$ & 0.145 & 0.984 & 0.989 \\
\hline $\mathbf{7}$ & 0.314 & $4.452 \times 10^{-5}$ & 0.005 & $1.135 \times 10^{-5}$ & 0.150 & 0.995 & 0.997 \\
\hline $\mathbf{1 0}$ & 0.356 & $6.936 \times 10^{-5}$ & 0.007 & $1.768 \times 10^{-5}$ & 0.224 & 0.997 & 0.998 \\
\hline
\end{tabular}




$$
\begin{aligned}
& \rho_{1, \max }=1060\left[\frac{\mathrm{kg}}{\mathrm{m}^{3}}\right], \\
& \rho_{1, \min }=1010\left[\frac{\mathrm{kg}}{\mathrm{m}^{3}}\right],
\end{aligned}
$$

and

$$
\rho_{1}^{r}=\operatorname{rand}(1010,1060)
$$

then,

$$
\rho_{1, \min } \leq \rho_{1}^{r} \leq \rho_{1, \max }
$$

Based on (18) - (20), the performance of NLMHE was evaluated for each density bound. The simulation results for the state variables, considering the same disturbances in the UI, are shown in Figure 5. From these results, it can be observed that the estimation using the highest possible limit of inlet density produces a higher estimation of slurry density. This increment in $\rho_{2}$ is compensated with lower values of $Q$ to satisfy the model equations because the pressure drop is considered constant, and therefore the system energy by slurry transport is (and should be) the same. From this fact, the only way to transport a slurry denser is by decreasing the flow and vice versa. The error indices obtained using a random value inside the feasible region for UI are presented in Table 4. Finally, from the estimation of slurry density and volumetric flow, the viscosity estimation is obtained based on (4) and the obtained result is shown in Figure 6.

According to the previous results, the estimation of the slurry properties always remains within the obtained interval from the possible values of $\rho_{1}$. Thus, the approximation by interval and the consideration of a random value for the slurry inlet density is a useful tool for the online estimation of slurry properties. In spite of random variation of the estimated values, to count with density and viscosity in real time is a valuable input for a closed-loop 
Table 4: Error indices for estimation based on the interval approximation of $\rho_{1}$.

\begin{tabular}{|c|c|c|c|c|c|c|}
\hline \multirow{2}{*}{ Unknown input } & \multicolumn{2}{|c|}{ RMS } & \multicolumn{2}{c|}{ RSD } & \multicolumn{2}{c|}{ IA } \\
\cline { 2 - 7 } & $\hat{\mathbf{Q}}\left[\frac{\mathrm{L}}{\mathrm{s}}\right]$ & $\hat{\boldsymbol{\rho}}_{\boldsymbol{2}}\left[\frac{\mathrm{kg}}{\mathrm{m}^{3}}\right]$ & $\hat{\mathbf{Q}}\left[\frac{\mathrm{L}}{\mathrm{s}}\right]$ & $\hat{\boldsymbol{\rho}}_{\mathbf{2}}\left[\frac{\mathrm{kg}}{\mathrm{m}^{3}}\right]$ & $\hat{\mathbf{Q}}\left[\frac{\mathrm{L}}{\mathrm{s}}\right]$ & $\hat{\boldsymbol{\rho}}_{\boldsymbol{2}}\left[\frac{\mathrm{kg}}{\mathrm{m}^{3}}\right]$ \\
\hline $\boldsymbol{\rho}_{\mathbf{1 , m a x}}$ & $1.918 \times 10^{-5}$ & 0.021 & $1.930 \times 10^{-5}$ & 0.235 & 0.955 & 0.971 \\
\hline $\boldsymbol{\rho}_{\mathbf{1}}^{r}$ & $7.571 \times 10^{-5}$ & 0.007 & $4.893 \times 10^{-5}$ & 0.674 & 0.865 & 0.943 \\
\hline $\boldsymbol{\rho}_{\mathbf{1}, \min }$ & $2.001 \times 10^{-5}$ & 0.032 & $5.118 \times 10^{-5}$ & 1.049 & 0.734 & 0.898 \\
\hline
\end{tabular}
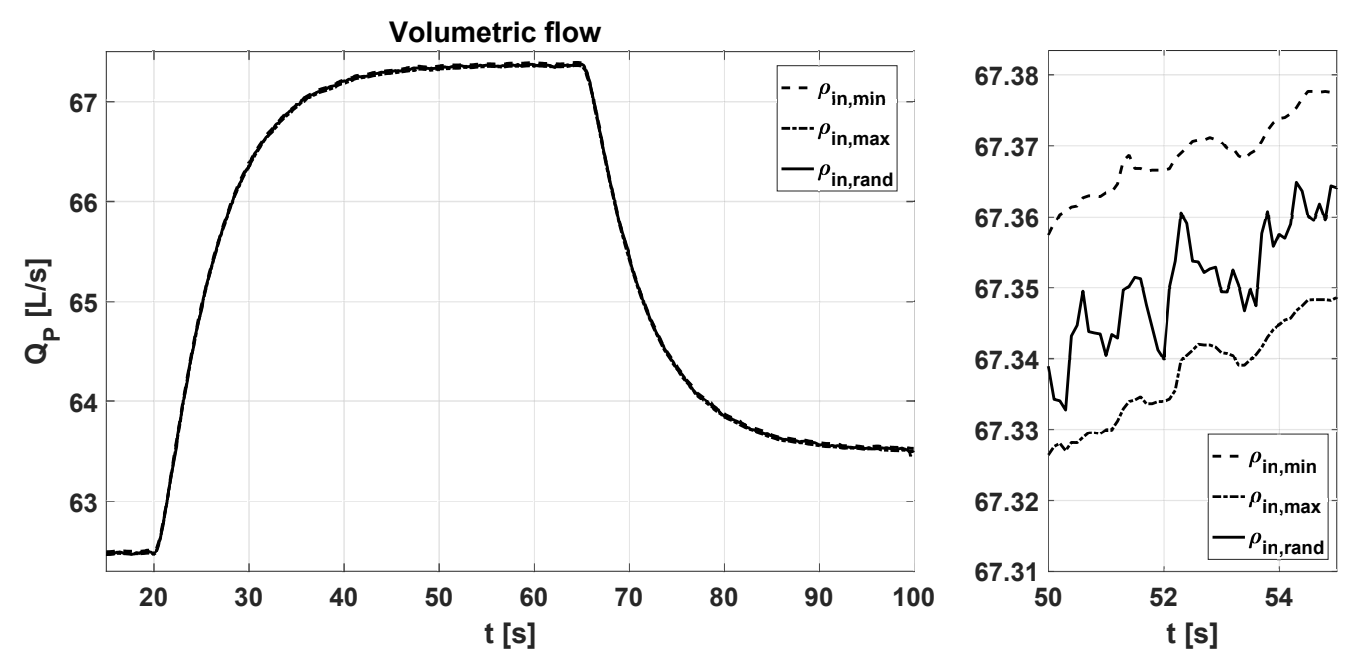

(a) Interval approximation for flow rate.
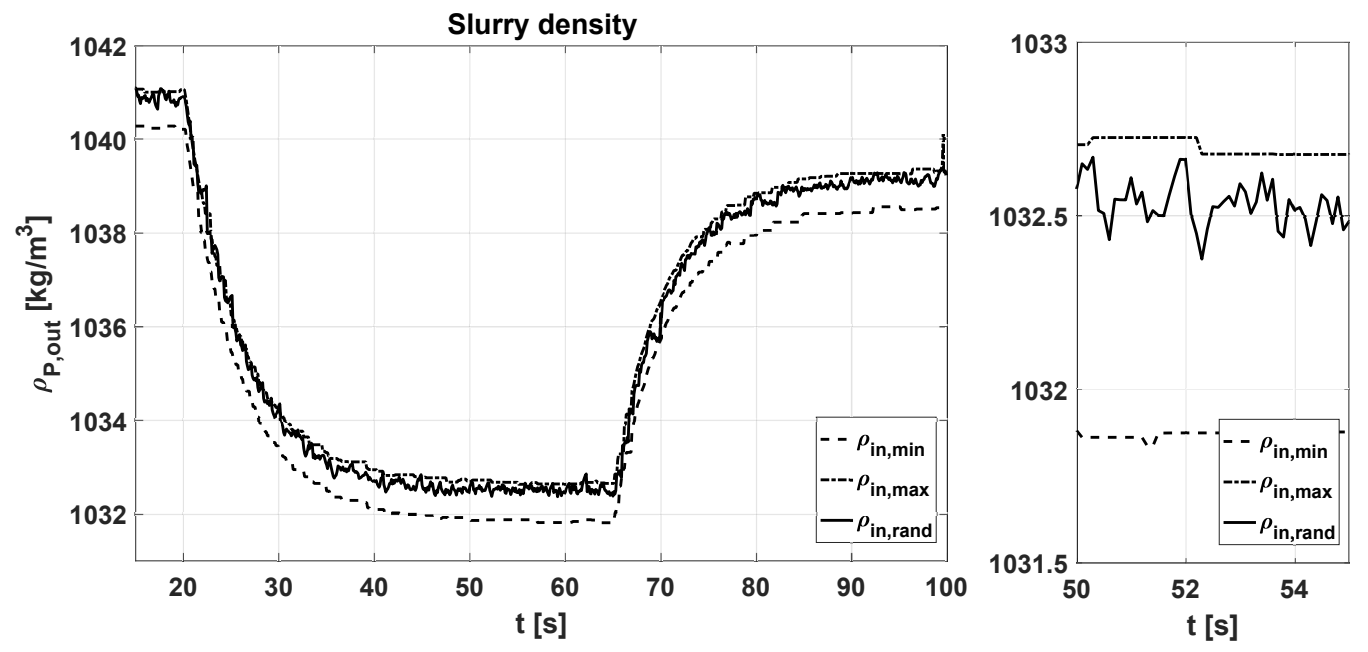

(b) Interval approximation for slurry density.

Figure 5: Simulation of the NLMHE with interval approximation of $\rho_{1}$ and $N=5$.

operation of separation units and other mineral processing equipment. 

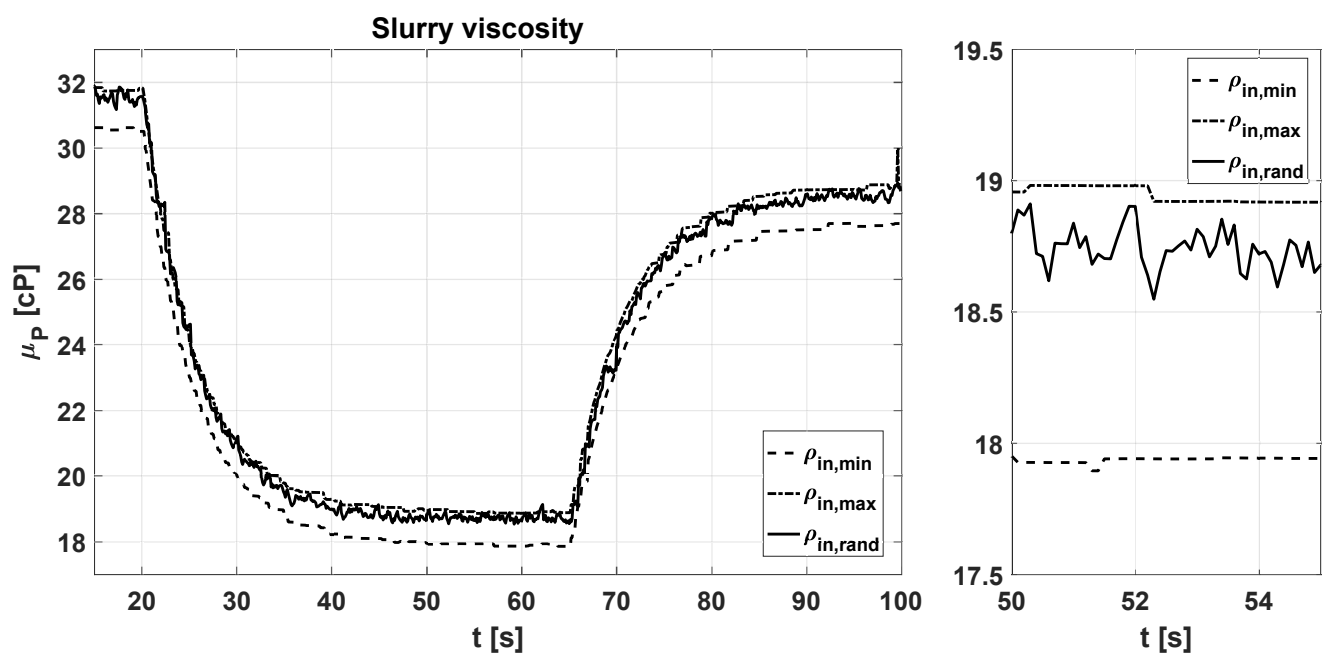

Figure 6: Simulation of NLMHE with interval approximation of $\rho_{1}$ and $N=5$.

\subsection{Comparison between the linear and nonlinear observers}

To compare the performance of the proposed NLMHE and the linear UIO presented in Ref. 16 , it was considered a random value of inlet density between an interval with mean value of $\rho_{1}=1040\left[\frac{\mathrm{kg}}{\mathrm{m}^{3}}\right]$. This latter takes into account that this value of $\rho_{1}$ corresponds to the stationary state used for designing the linear UIO. ${ }^{16}$ For comparing both estimators, the simulation conditions remained the same. From this, the same total time of simulation, the disturbances, and the noise for the measured variable was considered.

For the case of the linear UIO, the estimation is restricted to regions near to a steady state, because the design of the observer involves a linearization of the nonlinear model equations in (1) at a steady state of process. Besides, one transformation of the linearized model, to eliminate the terms that contain the UI, is implemented. ${ }^{16}$ Thus, this observer does not consider the model nonlinearities, and as the process moves away from the steady state over which it was linearized, the estimation error will be propagated and the estimation becomes worse.

The simulation results obtained for the linear UIO and the NLMHE are shown in Figure 7 and 8. For this case, and since disturbances are considered, the admissible interval for $\rho_{1}$ 

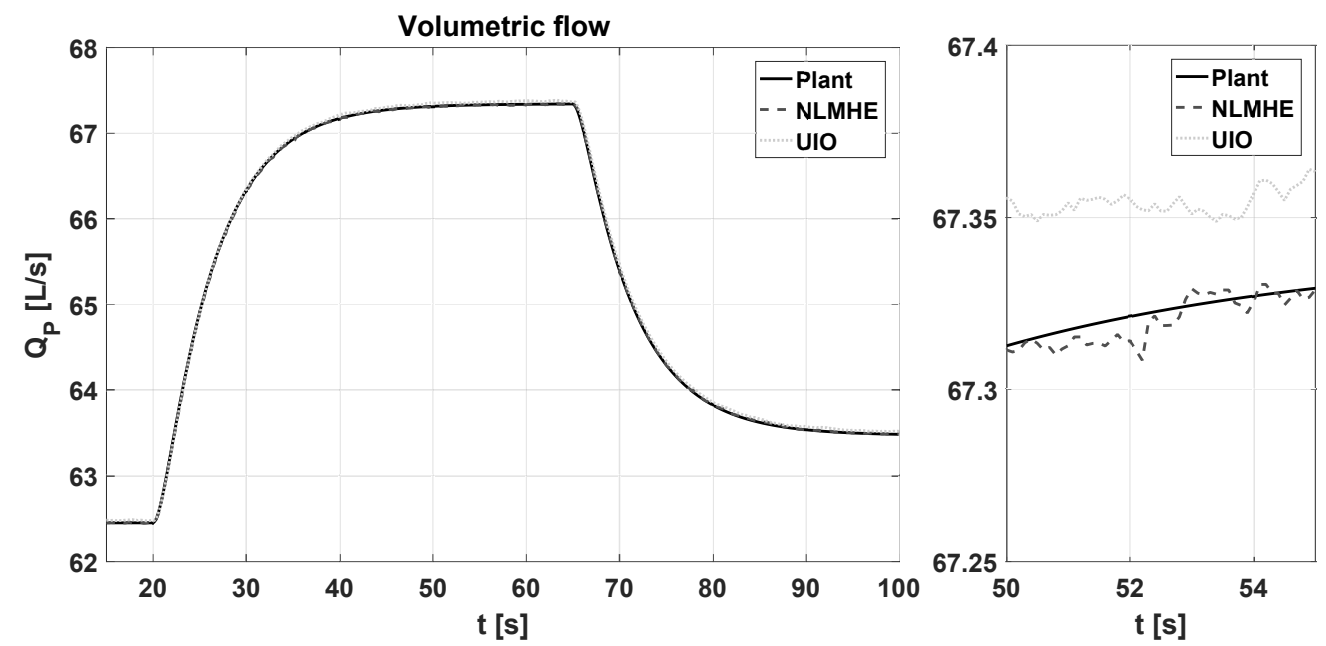

(a) Estimation of measured state using the NLMHE with $N=5$, and the linear UIO proposed in Ref. 16.
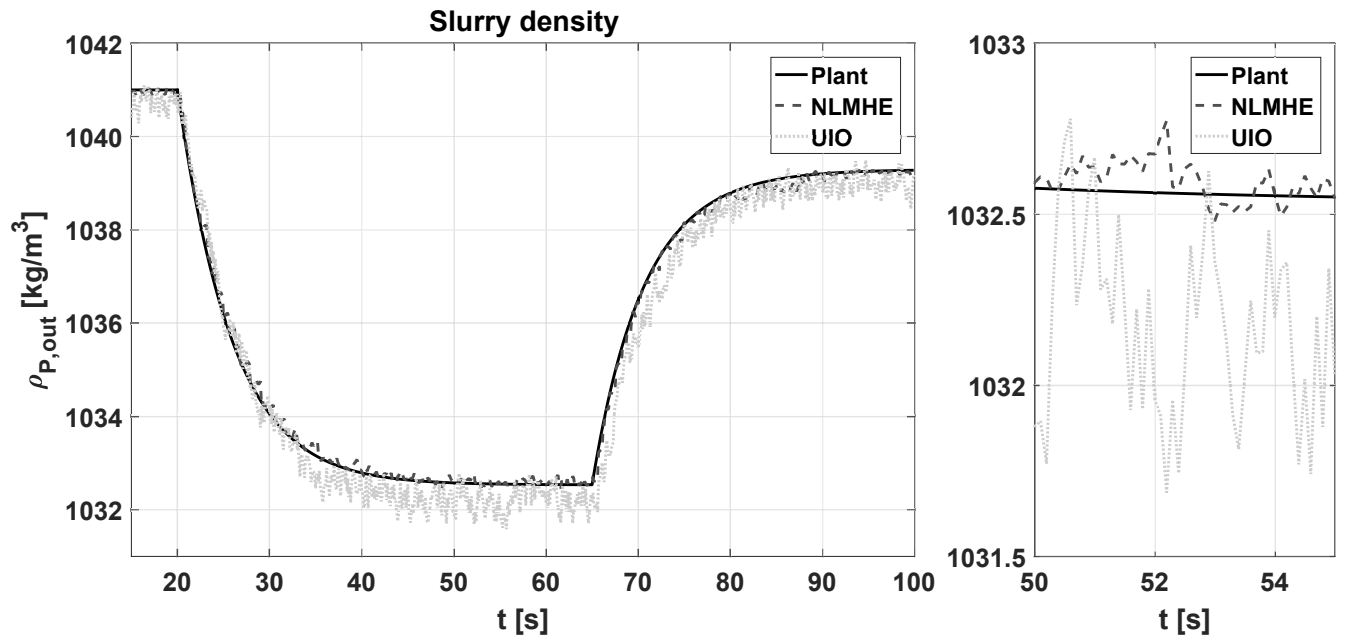

(b) Estimation of unmeasured state using the NLMHE with $N=5$, and the linear UIO proposed in Ref. 16.

Figure 7: Comparation of performance of NLMHE and UIO proposed in Ref. 16.

was set so that the value of steady state (for the UIO) corresponds to the mean value. In addition, the sampling time was the same $(\Delta t=0.1 \mathrm{~s})$ for both cases. In Table 5 , the error indices for the online estimation of measured and unmeasured variables is presented for both estimation structures: linear UIO and NLMHE.

According to the obtained results, the NLMHE shows a better online estimation of slurry properties than the linear version of UIO presented in Ref. 16. In addition, the NLMHE has error indices significantly lower than the UIO, both in the measured and 

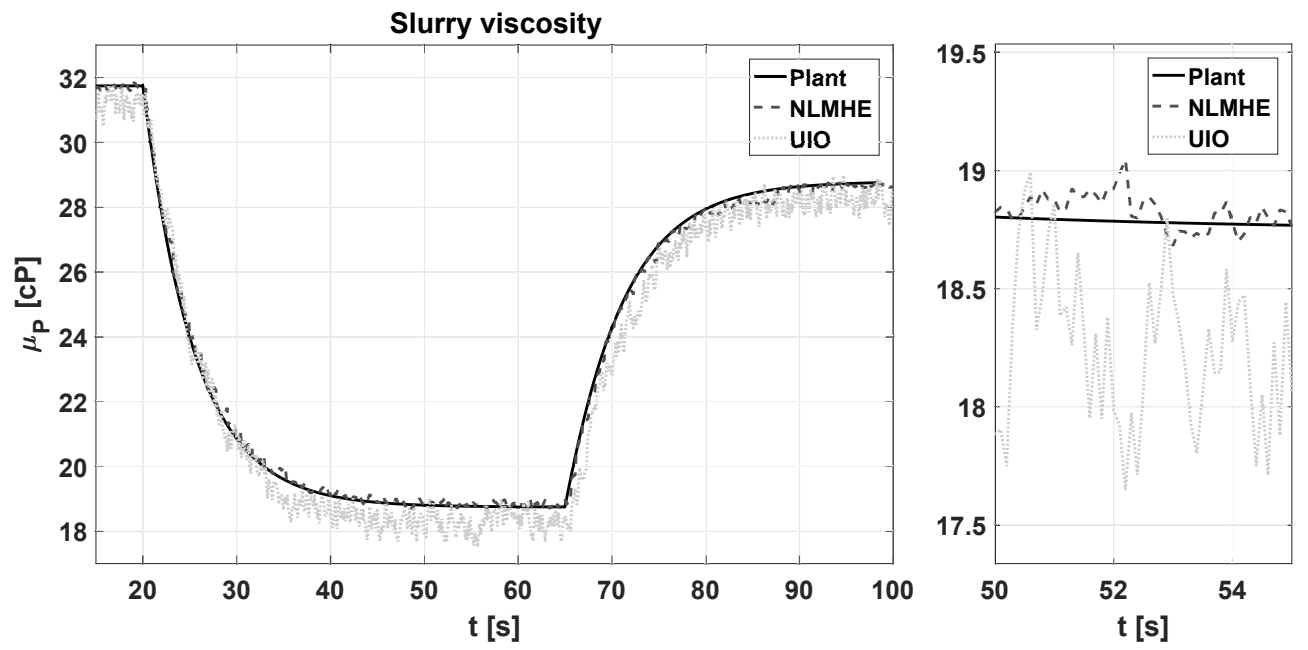

Figure 8: Viscosity estimation using the NLMHE with $N=5$, and the linear UIO proposed in Ref. 16.

unmeasured variables, and the computational time by iteration is lower when the model linearization is not required.

\section{Conclusions and future work}

In this paper, a proposal for online estimation of the properties of a mineral slurry flowing through a PFA is presented. The main advantage of this proposal is that, although it considers the disturbances in the process, it does not assume a specific dynamic for the unknown input. Taking into account that the estimator is designed by stating an optimization problem, which does not require any mathematical transformation of the model equations, the nonlinearities of the model are always considered.

From the proposed NLMHE, considering the unknown input as a random value, it is

Table 5: Error indices for estimation based on UIO and NLMHE.

\begin{tabular}{|c|c|c|c|c|c|c|}
\hline \multirow{2}{*}{ Estimator } & \multicolumn{2}{|c|}{ RMS } & \multicolumn{2}{c|}{ RSD } & \multicolumn{2}{c|}{ IA } \\
\cline { 2 - 7 } & $\hat{\mathbf{Q}}\left[\frac{\mathrm{L}}{\mathrm{s}}\right]$ & $\hat{\boldsymbol{\rho}}_{\mathbf{2}}\left[\frac{\mathrm{kg}}{\mathrm{m}^{3}}\right]$ & $\hat{\mathbf{Q}}\left[\frac{\mathrm{L}}{\mathrm{s}}\right]$ & $\hat{\boldsymbol{\rho}}_{\mathbf{2}}\left[\frac{\mathrm{kg}}{\mathrm{m}^{3}}\right]$ & $\hat{\mathbf{Q}}\left[\frac{\mathrm{L}}{\mathrm{s}}\right]$ & $\hat{\boldsymbol{\rho}}_{\mathbf{2}}\left[\frac{\mathrm{kg}}{\mathrm{m}^{3}}\right]$ \\
\hline UIO & $9.949 \times 10^{-5}$ & 0.0331 & $2.539 \times 10^{-5}$ & 0.435 & 0.989 & 0.846 \\
\hline NLMHE & $3.389 \times 10^{-5}$ & 0.004 & $8.618 \times 10^{-6}$ & 0.127 & 0.991 & 0.993 \\
\hline
\end{tabular}


possible to obtain the estimation of slurry properties without restricting the estimation to a particular operation point (or stationary state). Therefore, the online estimation can be performed covering all admissible values of the unknown inputs or disturbances affecting the process. Based on the historical data set of the measured and unmeasured variables, the PBSM, and the operating range for the slurry inlet density, the solution of the optimization problem in (14) yields a sequence of optimal values of the state variables from $T-N$ until $T$ of size equal to $N$.

Although only the measurements of the volumetric flow are considered in the cost function, the estimation of both the volumetric flow and the slurry density are performed with significantly lower estimation errors and small computational times using measured pressures into the model as known inputs. This latter even when noise is considered in the measured variable and independently of the inlet density. However, the estimation could be improved by considering other forms of the cost function. Therefore, some laboratory tests (done to determine slurry properties as a quality indicator, for example) could be included for updating the data set or the moving horizon, or as an asynchronous measurement in the cost function, in order to improve the optimization result.

Comparing the two estimators, the linear observer proposed in Ref. 16 and the nonlinear estimator proposed in this work (NLMHE), the state estimation using the NLMHE is better and faster than the estimation using the linear observer, even when the nonlinear estimation has been stated as an optimization problem. This fact is given since the linear version of the estimator proposed in Ref. 16 requires a sampling time $\Delta t=0.1 \mathrm{~s}$ for solving the differential equations, which involves many iterations, while the optimization problem considering in the moving horizon estimation method is not restricted to a specific sampling time except for computational burden limitations. However, in this case, the EKF implemented for the arrival cost is limited to a sampling time $\Delta t=0.1 \mathrm{~s}$ too. From this fact, the evaluation of more efficient alternatives for computing the arrival cost can be considered in order to reduce the computational time. 
Finally, from the proposed approach for the design of observers, based on the method of moving horizon estimation, it is possible to handle nonlinear systems of unknown inputs. This proposal is not restrictive for the model structure, but it requires information about the operational range of the unknown inputs. However, the proposed scheme was tested for systems with a number of UI equal to the number measured variables. An analysis for systems that do no satisfy this requirement must be done.

\section{Appendix}

According to Ref. 16, an algebraic equation for calculating the slurry viscosity of pseudoplastic fluids can be represented by the power law model

$$
\mu(t)=K_{\mu}(t)|\dot{\gamma}(t)|^{n(t)-1},
$$

where $\mu, \dot{\gamma}, K_{\mu}$ and $n$ are the viscosity, the shear rate, the consistency factor and the behavior index, respectively. In addition, for computing the parameters $K_{\mu}$ and $n$, two polynomial correlations in function of solids concentration have been proposed as follows:

$$
\begin{aligned}
& K(t)=981072 w_{v, \text { sol }}^{2}(t)-45397 w_{v, \text { sol }}(t)+583.01, \\
& n(t)=-269.87 w_{v, \text { sol }}^{3}(t)+104.09 w_{v, \text { sol }}^{2}(t)-12.009 w_{v, \text { sol }}(t)+0.6188 .
\end{aligned}
$$

So far, replacing (22) and (23) in (21), an expression for the viscosity, in function of the solids concentration is obtained. However, the viscosity should also consider the flow conditions in the pipeline. Therefore, the shear rate $\dot{\gamma}$ in (21) is computed taking into account the type of duct to obtain an expression of the viscosity that considers the real flow conditions. For non-Newtonian fluids, Madlener et al. in 2009 proposed the following expression for the shear rate: 


$$
\dot{\gamma}(t)=\left(\frac{3 n(t)+1}{4 n(t)}\right) \frac{8 \bar{u}(t)}{D_{T}}
$$

where $\bar{u}$ and $D_{T}$ are the average velocity in the line and the diameter of the pipe, respectively. Finally, the expression for the slurry viscosity is given by:

$$
\mu(t)=K_{\mu}(t)\left[\left(\frac{3 n(t)+1}{4 n(t)}\right) \frac{8 \bar{u}(t)}{D_{T}}\right]^{n(t)-1} .
$$

\section{References}

(1) Witczak, M. Fault Diagnosis and Fault-Tolerant Control Strategies for Non-Linear Systems: Analytical and Soft Computing Approaches, 1st ed.; Springer International Publishing: Switzerland, 2014; pp $19-56$.

(2) Rincon, A.; Angulo, F.; Olivar, G. Control of an anaerobic digester through normal form of fold bifurcation. Journal of Process Control 2009, 19, 1355 - 1367.

(3) Besançon, G. Nonlinear observers and applications, 1st ed.; Springer: New York, 2007.

(4) Hodouin, D. Methods for automatic control, observation, and optimization in mineral processing plants. Journal of Process Control 2011, 21, 211 - 225.

(5) Pertew, A. M.; Marquez, H. J.; Zhao, Q. Design of unknown input observers for Lipschitz nonlinear systems. Proceedings of the American Control Conference. 2005; pp $4198-4203$.

(6) Koenig, D. Observer design for unknown input nonlinear descriptor systems via convex optimization. IEEE Transactions on Automatic Control 2006, 51, 1047 - 1052.

(7) Barbot, J.-P.; Boutat, D.; Floquet, T. An observation algorithm for nonlinear systems with unknown inputs. Automatica 2009, 45, 1970 - 1974. 
(8) Garcia-Tirado, J.; Botero, H.; Angulo, F. A new approach to state estimation for uncertain linear systems in a moving horizon estimation setting. International Journal of Automation and Computing 2016, 13, 653 - 664.

(9) Alamir, M. On Adaptive Measurement Inclusion Rate in Real-Time Moving-Horizon Observers. IFAC Proceedings Volumes 2014, 47, 552 - 557.

(10) ühl, P. K.; Diehl, M.; Kraus, T.; öder, J. P. S.; Bock, H. G. A real-time algorithm for moving horizon state and parameter estimation. Computers $\mathcal{E}$ Chemical Engineering 2011, 35, $71-83$.

(11) Ji, L.; Rawlings, J. B. Application of MHE to large-scale nonlinear processes with delayed lab measurements. Computers \& Chemical Engineering 2015, 80, 63 - 72.

(12) Chhabra, R.; Richardson, J. In Non Newtonian Flow and Applied Rheology (Second Edition), 2nd ed.; Chhabra, R., Richardson, J., Eds.; Butterworth-Heinemann: Oxford, 2008; pp $56-109$.

(13) Botero, H. Formalismo para la síntesis de sensores virtuales basados en un modelo maestro de base fenomenológica. Ph.D. thesis, Universidad Nacional de Colombia Departamento de procesos y energía, 2010.

(14) Ali, J. M.; Hoang, N. H.; Hussain, M.; Dochain, D. Review and classification of recent observers applied in chemical process systems. Computers and Chemical Engineering 2015, 76, $27-41$.

(15) Benretem, A.; Benidir, M.; Chaib, R. Factors influencing slurry rheology. World Pumps 2010, 2010, $30-32$.

(16) Diaz C., J. L. The use of phenomenological based semi-physical models as virtual sensors for density and viscosity of mineral slurries. M.Sc. thesis, Universidad Nacional de Colombia - Facultad de Minas, 2017. 
(17) Hoyos, E.; López, D.; Alvarez, H. A phenomenologically based material flow model for friction stir welding. Materials \& Design 2016, 111, 321 - 330.

(18) Hooper, W. B. The two K method predics head losses in pipe and fittings. Chemical Engineering 1981, 96 - 100.

(19) Schorle, B. J.; Churchill, S. W.; Shacham, M. Comments on: An Explicit Equation for Friction Factor in Pipe. Industrial and Engineering Chemistry 1980, 19, 228 - 228.

(20) Madlener, K.; Frey, B.; Ciezki, H. K. Generalized Reynolds number for non-newtonian fluids. Progress in Propulsion Physics. 2009.

(21) Raff, T.; Lachner, F.; Allgöwer, F. A Finite Time Unknown Input Observer For Linear Systems. 14th Mediterranean Conference on Control and Automation. 2006; pp 1-5.

(22) Tian, J.; Ma, S. Unknown input observer design for one-sided Lipschitz nonlinear continuous-time singular Markovian jump systems. 12th World Congress on Intelligent Control and Automation (WCICA). 2016; pp 1920 - 1925.

(23) Liu, X.; Gao, Z. Novel unknown input observer for fault estimation of gas turbine dynamic systems. IEEE 13th International Conference on Industrial Informatics (INDIN). 2015; pp 562-567.

(24) Boegli, M. Real-Time Moving Horizon Estimation for Advanced Motion Control. Application to Friction State and Parameter Estimation. Ph.D. thesis, Faculty of Engineering Science - KU Leuven, 2014.

(25) Sui, D.; Nybo, R.; Hovland, S.; Johansen, T. A Moving Horizon Observer for Estimation of Bottomhole Pressure during Drilling. IFAC Proceedings Volumes 2012, 45, 145 150.

(26) Rao, C. V. Moving Horizon Strategies for the Constrained Monitoring and Control of 
Nonlinear Discrete Time Systems. Ph.D. thesis, Department of Chemical Engineering - University of Wisconsin - Madison, 2000.

(27) Ramalingam, M. K. Moving Horizon Estimation with Dynamic Programming. M.Sc. thesis, Department of Chemical and Biomedical Engineering - Cleveland State University, 2013.

(28) Diehl, M.; Ferreau, H. J.; Haverbeke, N. Nonlinear Model Predictive Control: Towards New Challenging Applications, 1st ed.; Springer: Berlin, Heidelberg, 2009. 